\title{
Avaliação de três métodos de obtenção do índice de área foliar para cultura da soja
}

\author{
Ana Laura Pereira SOUZA ${ }^{1 *}$, Marcelo Marques COSTA $^{1}$, Darly Geraldo de SENA JUNIOR ${ }^{1}$, \\ Rogério Borges de Oliveira PAZ ${ }^{1}$
}

${ }^{1}$ Universidade Federal de Goiás, Regional Jataí, Jataí, GO, Brasil.

E-mail: marcelo.marques.costa@gmail.com

Recebido em outubro/2018; Aceito em fevereiro/2019.

\begin{abstract}
RESUMO: O Índice de área foliar (IAF) representa a eficiência na interceptação e utilização da radiação solar pelas plantas. O IAF pode ser obtido de forma direta, determinando-se a área foliar e área ocupada pela planta. Entretanto, métodos indiretos são comumente utilizados para sua estimativa devido a necessidade de praticidade. O objetivo deste trabalho foi avaliar a equivalência das estimativas do IAF obtidas por meio de um ceptômetro, pelo método de discos e utilizando imagens digitais. Para tanto, foi conduzido um experimento com a cultura da soja, no delineamento em blocos casualizados, com variação da densidade de plantas, para comparar os valores de IAF obtidos pelos diferentes métodos aos 34 dias após a semeadura (DAS). A comparação foi feita por meio dos coeficientes de equações lineares ajustadas entre os resultados obtidos. O método do disco e do ceptômetro, apresentaram uma boa equivalência entre seus valores. Entretanto, o método que utiliza imagens superestimou o IAF, não apresentando uma boa equivalência de seus valores aos do método de discos e ao ceptômetro.
\end{abstract}

Palavras-chave: ceptômetro, discos foliares, imagens digitais.

\section{Evaluation of three obtainment methods of foliar area index for soybean crop}

\begin{abstract}
The leaf area index (LAI) represents the interception efficiency and use of solar radiation by plants. IAF can be obtained directly, determining the leaf area and area occupied by the plant. However, indirect methods are commonly used for their estimation because for practicality. The objective of this work was to evaluate the equivalence of the LAI estimates obtained with a ceptometer, using the disc method and digital images. A experiment was conducted with soybean crop, in a randomized block design with plant density variation to compare the LAI values obtained by the different methods at 34 days after sowing (DAS). The comparison was made through the coefficients of linear equations adjusted between the results obtained. The disc and the ceptometer method presented a good equivalence between their values. However, the imaging method overestimated the LAI, not presenting a good equivalence of its values to those of the disc method and the ceptometer.
\end{abstract}

Keywords: ceptometer, leaf discs, digital images.

\section{INTRODUÇÃO}

$\mathrm{O}$ aproveitamento da radiação solar pelos vegetais, entre outros fatores, é de grande importância para obtenção de um bom resultado em seu desenvolvimento. A produtividade das culturas, entre elas a soja, é determinada pela capacidade de interceptação da radiação solar pelas plantas e mensurada por meio do índice de área foliar (IAF) e a conversão desta radiação em matéria seca através do processo fotossintético (ZANON et al., 2015). O IAF é definido como a relação entre a área foliar de uma planta e a área de solo ocupada pela mesma (HEIFFIG et al., 2006).

$\mathrm{O}$ arranjo e a população de plantas podem influenciar o desenvolvimento e a produção das culturas, pois altera a disponibilidade dos recursos água, luz e nutrientes para cada planta. Entretanto, as plantas de soja podem alterar sua arquitetura por meio de ramificações, elevando o número de grãos produzidos por planta (FERREIRA et al., 2018) e consequentemente o número de folhas e a área foliar de cada planta.

Portanto, a área foliar (AF) e o Índice de Área Foliar (IAF) são bons indicativos do potencial de produtividade, no qual a energia luminosa interceptada é convertida em energia química na forma de fotoassimilados (FAVARIN et al., 2002).
Sendo assim, é esperado que nas áreas em se observa variação espacial do IAF seja verificada variabilidade semelhante na produtividade das culturas. Scarpari; Beauclair (2008) observaram na cultura da cana-de-açúcar grande variação espacial nos valores de IAF e Brix, inversamente proporcionais devido à disponibilidade hídrica. Os autores concluem que essa variabilidade deve ser considerada para a estimativa da produtividade da cana-de-açúcar, minimizando erros. Além disso, Shafian et al. (2018) observaram elevada correlação entre índices espectrais, IAF e produtividade do sorgo, indicando sua aplicabilidade para coleta de dados durante o ciclo da cultura.

Alguns métodos para obtenção do IAF podem ser trabalhosos e destrutivos, apesar de sua eficiência. Os métodos destrutivos exigem a retirada das folhas, o que pode comprometer a parcela. Já nos métodos não destrutivos não há necessidade de remoção das folhas, garantindo a integridade das plantas (ADAMI et al., 2008).

O IAF pode ser determinado ainda por meio de técnicas de processamento de imagens (ADAMI et al. 2008), porém esse método é destrutivo e trabalhoso, pois exige a retirada de todas as folhas para a determinação da área foliar. Outro método, também destrutivo e trabalhoso de obter o IAF, tem como base 
a estimativa da $\mathrm{AF}$ utilizando discos foliares com área conhecida (ADAMI et al., 2008). Já o ceptômetro possibilita um método não destrutivo de estimar o IAF, utilizando a medida indireta da radiação solar interceptada pelo dossel das plantas. Segundo Galzerano et al. (2012), a radiação fotossinteticamente ativa registrada pelo ceptômetro corresponde positivamente ao índice de área foliar.

Já foi observada também uma relação significativa entre o IAF e a matéria seca da parte aérea (MSPA) de plantas de soja, como no trabalho de Balbinot Junior et al. (2016). Assim, esses autores indicaram que é possível estimar o IAF a partir de MSPA, facilitando os trabalhos quando não há métodos disponíveis para determinação do IAF. Além disso, se for possível determinar o IAF por método não destrutivo, pode-se estimar a MSPA sem a destruição de plantas.

O objetivo do trabalho foi avaliar a equivalência na determinação do IAF, na cultura da soja, utilizando o método de processamento de imagens, método de discos foliares e método de ceptômetro.

\section{MATERIAL E MÉTODOS}

$\mathrm{O}$ experimento foi conduzido na área experimental da Universidade Federal de Goiás, Regional Jataí, no município de Jataí - GO, durante o ano agrícola 2016/2017. O solo da área experimental é classificado como Latossolo Vermelho distroférrico. A adubação foi realizada manualmente a lanço e as doses utilizadas seguiram as recomendações para elevadas produtividades. Além disso, o manejo fitossanitário foi realizado conforme recomendação para a cultura da soja na região.

O cultivar de soja utilizado foi a M7739 e o delineamento experimental adotado foi o de blocos ao acaso com quatro repetições e cinco tratamentos, nos quais se variou a densidade de plantas. As populações adotadas nos tratamentos, no momento da semeadura, foram 6,$9 ; 10,1 ; 14,0 ; 17,7$ e 21,3 sementes $\mathrm{m}^{-1}$.e espaçamento entre linhas de $0,45 \mathrm{~m}$. Com a variação da população de plantas foi obtida uma amplitude de variação no índice de área foliar, determinado em um único momento durante o ciclo da cultura. As parcelas foram implantadas na área experimental por meio de uma semeadora mecânica de cinco linhas da marca Tatu Marchesan.

\subsection{IAF utilizando ceptômetro}

Foi utilizado um ceptômetro modelo LP-80 (AccuPAR LP-80, Decagon Devices, Pullman, Washington, EUA). Esse equipamento mede a radiação fotossinteticamente ativa e a partir dessa calcula a o IAF por meio de equações no software do aparelho. Foram realizadas cinco subamostras para compor a média do IAF de cada parcela. As leituras foram realizadas em condição de céu limpo, sem presença de nuvens. Foi utilizado um sensor externo, mantido acima do dossel da cultura e uma barra com sensores que foi posicionado abaixo do dossel da cultura. Pela diferença na incidência luminosa entre os sensores o equipamento calcula a interceptação luminosa e estima o IAF.

\subsection{IAF utilizando imagens digitais}

Para o método de imagem foi recolhida uma amostra composta por três plantas de cada parcela. Essas amostras foram levadas imediatamente para um ambiente fresco, onde foram destacadas todas as folhas, que então foram colocadas sobre uma folha de papel com as dimensões $0,82 \mathrm{~m}$ x 0,62 m. Com todas as folhas distribuídas sobre o papel, obteve-se uma imagem com a câmera digital posicionada verticalmente acima do mesmo. Este processo se repetiu em todas as amostras de cada parcela. Por meio do programa computacional SisCob (JORGE; SILVA, 2009) as imagens foram classificadas, obtendo-se as porcentagens da área ocupada pelas folhas de soja e pelo papel. A partir dos valores percentuais obtidos da classificação foi determinado a AF pela Equação 1 e o IAF pela Equação 2.

$$
\mathrm{AF}_{\text {imagem }}=\frac{\mathrm{f} * 5084}{3 *(\mathrm{f}+\mathrm{p})}
$$

em que: $\mathrm{AF}=$ Área foliar média de uma planta $\left(\mathrm{cm}^{2}\right) ; \mathrm{f}=$ Porcentagem de folhas na imagem (decimal); $\mathrm{p}=$ Porcentagem de papel na imagem (decimal).

$$
\mathrm{IAF}_{\text {imagem }}=\frac{\mathrm{AF}_{\text {imagem }}}{\mathrm{AP}}
$$

em que: $\mathrm{IAF}_{\text {imagem }}=$ Índice de área foliar obtida a partir do processamento de imagens; $\mathrm{AF}_{\text {imagem }}=$ Área foliar média de uma planta obtida pelo processamento das imagens $\left(\mathrm{cm}^{2}\right)$; AP $=$ Área ocupada por uma planta $\left(\mathrm{cm}^{2}\right)$.

\subsection{IAF utilizando discos}

O método dos discos, para obtenção do IAF, consiste em estimar a área de folhas a partir da massa de uma área conhecida de folhas. Neste trabalho foi utilizado um cilindro com seção de $3,04 \mathrm{~cm}^{2}$, sendo utilizado para retirar cinco discos foliares de cada amostra de folhas. As amostras de folhas consistiam em todas as folhas de três plantas. Foi determinada a massa dos cinco discos foliares e de todas as folhas, para determinar a AF conforme a Equação 3, e o IAF conforme a Equação 4.

$$
\mathrm{AF}_{\text {discos }}=\frac{5,07 * \mathrm{Mt}}{\mathrm{Md}}
$$

em que: $\mathrm{AF}_{\text {discos }}=$ Área foliar média de uma planta obtida pelo método dos discos $\left(\mathrm{cm}^{2}\right)$; $\mathrm{Mt}=$ Massa úmida de todas as folhas de três plantas (g); Md = Massa úmida de cinco discos (g).

$$
\mathrm{IAF}_{\text {discos }}=\frac{\mathrm{AF}_{\text {discos }}}{\mathrm{AP}}
$$

em que: IAF = Índice de Área foliar obtido pelo método dos discos.; $\mathrm{AF}=$ Área foliar média de uma planta $\left(\mathrm{cm}^{2}\right) ; \mathrm{AP}=$ Área ocupada por uma planta $\left(\mathrm{cm}^{2}\right)$.

\subsection{Comparação entre os métodos de determinação do IAF}

Para comparar os métodos foram ajustadas equações lineares entre os IAF estimados pelo ceptômetro e método dos discos, ceptômetro e método das imagens e entre o método das imagens e o método dos discos. Foi avaliado o coeficiente de determinação $\mathrm{R}^{2}$ como indicador da dispersão dos valores entre dois métodos. O coeficiente angular e o intercepto foram utilizados para indicar tendências de superestimativa ou subestimativa entre os métodos.

\section{RESULTADOS}

Pelo ajuste da regressão linear entre os valores de IAF estimado, os métodos de ceptômetro e disco apresentaram boa correspondência (Figura 1), porém com dispersão entre os valores obtidos pelos dois métodos. Na Figura 2 é apresentada a comparação entre os métodos do ceptômetro e das imagens 
enquanto na Figura 3 é apresentada a comparação entre os métodos dos discos e das imagens. $\mathrm{Na}$ Tabela 1 são apresentados os parâmetros e o $\mathrm{R}^{2}$ das equações ajustadas.

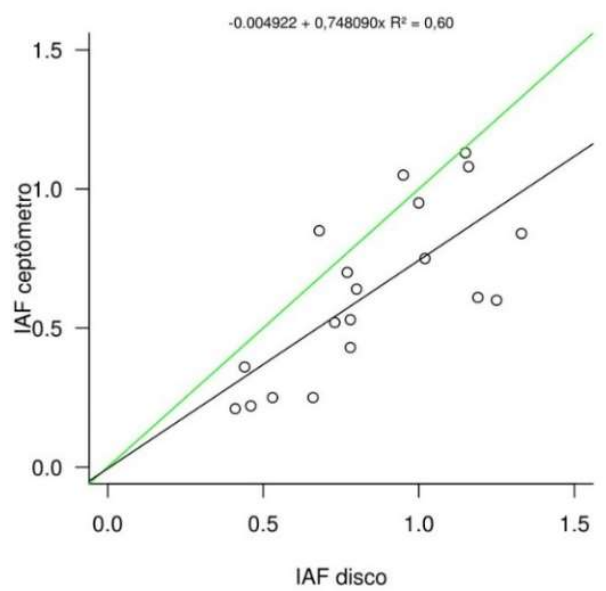

Figura 1. Modelo linear comparando o IAF obtido pelos métodos de ceptômetro e disco.

Figure 1. Linear model comparing the LAI obtained by the ceptometer and disk methods.

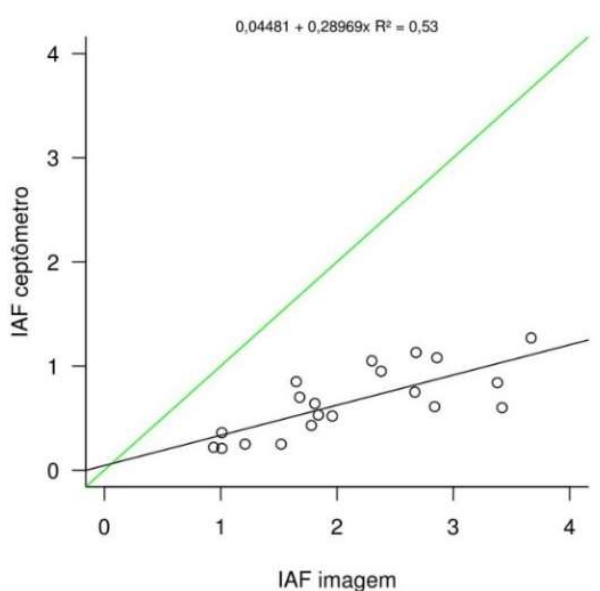

Figura 2 - Modelo linear comparando o IAF obtido pelos métodos de ceptômetro e imagens.

Figure 2 - Linear model comparing the LAI obtained by ceptometer and images methods.

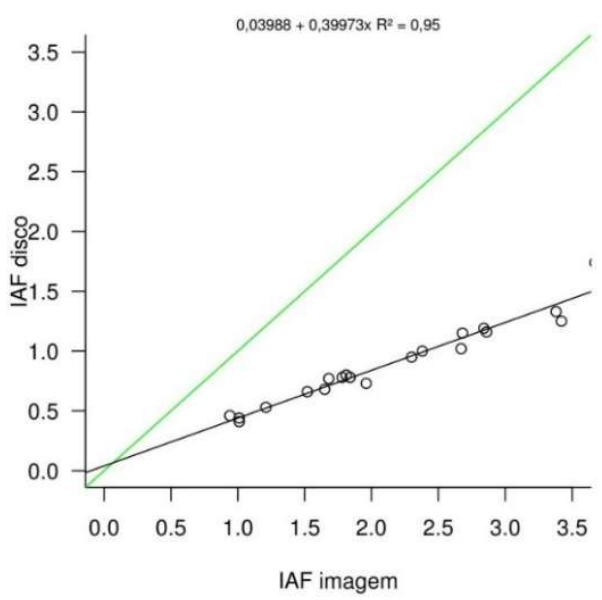

Figura 3 - Modelo linear comparando o IAF obtido pelos métodos de disco e imagens

Figure 3 - Linear model comparing the LAI obtained by disk and images methods.
Tabela 1. Valores de coeficiente angular (a), intercepto (b) e coeficiente de determinação $\left(\mathrm{R}^{2}\right)$ das comparações entre os métodos de obtenção do IAF.

Table 1. Values of angular coefficient (a), intercept (b) and determination coefficient $\left(\mathrm{R}^{2}\right)$ of the comparisons between the LAI obtaining methods.

\begin{tabular}{lrcc}
\hline \multicolumn{1}{c}{ Comparação } & $\mathrm{a}$ & $\mathrm{b}$ & $\mathrm{R}^{2}$ \\
\hline Ceptômetro x Disco & $-4,92 \mathrm{E}-03$ & 0,75 & 0,60 \\
Ceptômetro x Imagens & $4,48 \mathrm{E}-02$ & 0,29 & 0,53 \\
Disco x Imagens & $3,98 \mathrm{E}-02$ & 0,40 & 0,95 \\
\hline
\end{tabular}

Em uma perfeita correspondência entre os métodos, o coeficiente angular (a) e o coeficiente de determinação $\left(\mathrm{R}^{2}\right)$ teriam o valor um e o intercepto (b) valor zero. Em termos de intercepto, as três comparações apresentaram b bem próximo de zero, com destaque para a regressão entre os valores observados nos métodos do ceptômetro e do disco.

Em termos de coeficiente angular (a) a melhor equivalência entre métodos foi também do ceptômetro e do disco, com valor de aproximadamente 0,75. As demais comparações, do ceptômetro com as imagens e do disco com imagens apresentaram valores muito baixos, indicando uma superestimativa do método das imagens em comparação com os outros métodos.

Avaliando-se o $\mathrm{R}^{2}$, destaca-se a comparação do método do disco e das imagens, com valor de 0,95 , indicando que apesar da tendência de superestimar os valores pelo método das imagens há um bom ajuste entre os valores obtidos por esse e o método dos discos foliares.

\section{DISCUSSÃO}

Os resultados do coeficiente das regressões indicaram que o método de processamento de imagens superestimou os valores do IAF em relação aos demais métodos avaliados. Entretanto, no trabalho de Adami et al., 2008, o método de imagens digitais apresentou boa correspondência com o integrador de área foliar, sem maiores tendências de superestimativa. No trabalho citado, o resultado do processamento de imagens no programa computacional Spring apresentou melhores resultados que o método de dimensões foliares.

Os métodos de disco e do ceptômetro, são utilizados em muitos trabalhos científicos e referidos como métodos de boa representatividade, porém os resultados são um pouco discrepantes. Toebe et al. (2010) concluíram, com base nos coeficientes de correlação linear de Pearson e de Spearman, que os métodos de discos foliares e de fotos digitais são concordantes. Por outro lado, Tavares-Júnior et al. (2002) observaram uma tendência de superestimar os valores de área foliar quando se utiliza o método de discos foliares em relação ao integrador de área foliar LI-COR.

Souza et al. (2012) avaliando a correlação entre os valores obtidos pelo método dos discos e por análise de imagens para a cultura da melancia consideraram boa a correlação, com resultados superiores ao integrador de área foliar.

Entretanto, a forma utilizada neste trabalho para obter a área foliar por meio das imagens apresenta características que podem favorecer a superestimativa do IAF. Por exemplo, foram utilizadas todas as folhas, mesmos as bem jovens e, além disso, as imagens apresentam distorções devido ao ângulo em que foram obtidas (Figura 4). O programa computacional utilizado para classificação das imagens não apresenta ferramentas que permitem avaliar a exatidão da 
classificação. Contudo, pela avaliação visual das imagens classificadas considerou-se que houve uma boa concordância.

Supõe-se que as regiões de transição entre os pixels de folha e os pixels de papel possam ter sido classificadas como folha, aumentando a área de cada folha. Essa hipótese explicaria o fato de a superestimativa do IAF ser maior para as amostras com mais folhas, pois o incremento de pixels de folhas seria proporcional ao perímetro total das mesmas. Dessa forma, acredita-se que a classificação utilizando imagens com maior resolução seja uma forma de amenizar esse problema.

As diferenças nas características dos softwares utilizados e principalmente a forma de obtenção das imagens com reflexos na exatidão da classificação podem ser os motivos para os resultados discrepantes observados na literatura.

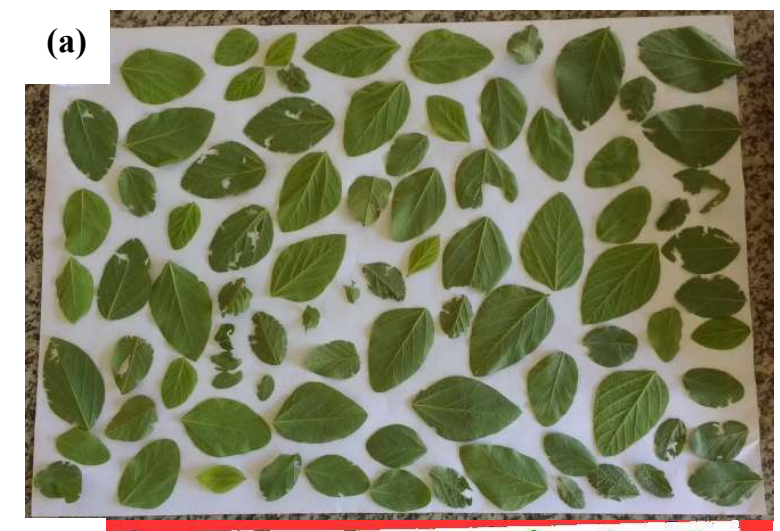

(b)

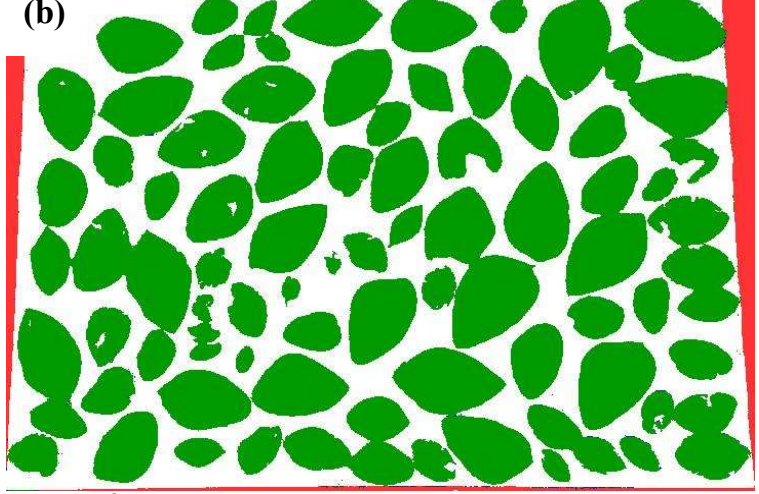

Figura 4. Área Foliar obtida por meio do programa SisCob: (a) Imagem original; (b) Imagem após a classificação.

Figure 4. Foliar area obtained using the SisCob software: (a) Original image; (b) Classified image.

\section{CONCLUSÕES}

O IAF obtido por imagens foi superestimado e não apresentou uma boa equivalência com o IAF obtido por ceptômetro e pelo método do disco.

O IAF obtido pelo método do disco e por ceptômetro apresentaram uma boa equivalência de seus valores.

\section{REFERÊNCIAS}

ADAMI, M.; HASTENREITER, F. A.; FLUMIGNAN, D. L.; FARIA, R. T. Estimativa de área de folíolos de soja usando imagens digitais e dimensões foliares. Bragantia, Campinas, v. 67, n. 4, p. 1053-1058, 2008. DOI: http://dx.doi.org/10.1590/S0006-87052008000400030

BALBINOT JUNIOR, A. O.; PROCOPIO, S. O. NEUMAIER, N.; FERREIRA, A. S.; WERNER, F.; DEBIASI, H.; FRANCHINI, J. C.; Semeadura cruzada, espaçamento entre fileiras e densidade de semeadura influenciando o crescimento e a produtividade de duas cultivares de soja. Revista de Ciências Agroveterinárias, Lages, v. 15, n. 2, p. 83-93, 2016. DOI: http://dx.doi.org/10.5965/223811711522016083

FAVARIN, J. L.; DOURADO NETO, D.; GARCÍA, A. G.; VILLA NOVA, N. A.; FAVARIN, M. G. G. V. Equações para a estimativa do índice de área foliar do cafeeiro. Pesquisa Agropecuária Brasileira, Brasília, v. 37, n. 6, p. 769-773, 2002. DOI: http://dx.doi.org/10.1590/S0100204X2002000600005

FERREIRA, A. S.; BALBINOT JUNIOR, A. A.; WERNER, F.; FRANCHINI, J. C.; ZUCARELI, C. Soybean agronomic performance in response to seeding rate and phosphate and potassium fertilization. Revista Brasileira de Engenharia Agrícola e Ambiental, v. 22, n. 3, p. 151157, 2018. DOI: http://dx.doi.org/10.1590/18071929/agriambi.v22n3p151-157

GALZERANO, L.; MALHEIROS, E. B.; MORGADO, E.S.; RUGGIERI, A. C. Interceptação de luz e índice de área foliar em relação a altura do dossel de gramíneas forrageiras. Nucleus Animalium, v. 4, n. 2, p. 11-18, 2012. DOI: http://dx.doi.org/ 10.3738/1982.2278.742

HEIFFIG, L. S.; CÂMARA, G. M. S.; MARQUES, L. A.; PEDROSO, D. B.; PIEDADE, S. M. S. Fechamento e índice de área foliar da cultura da soja em diferentes arranjos espaciais. Bragantia, Campinas, v. 65, n. 2, p. 285-295, 2006. DOI: http:// dx.doi.org/10.1590/S000687052006000200010

JORGE, L. A. C.; SILVA, D. J. C. B. SisCob: manual de utilização. São Carlos: Embrapa Instrumentação Agropecuária, 2009. 18 p.

SCARPARI, M. S.; BEAUCLAIR, E. G. F. Variação espaçotemporal do índice de área foliar e brix em cana-de-açúcar. Bragantia, Campinas, v. 67, n. 1, p. 35-41, 2008. http://dx.doi.org/10.1590/S0006-87052008000100004

SHAFIAN, S.; RAJAN, N.; SCHNELL, R.; BAGAVATHIANNAN, M.; VALASEK, J.; SHI, Y; OLSENHOLLER, J. Unmanned aerial systems-based remote sensing for monitoring sorghum growth and development. Plos One, n. 13, 2018. https://doi.org/10.1371/journal.pone.0196605

SOUZA, M. S.; ALVES, S. S. V.; DOMBROSKI, J. L. D.; FREITAS, J. D. B.; AROUCHA, E. M. M. Comparação de métodos de mensuração de área foliar para a cultura da melancia. Pesquisa Agropecuária Tropical, Goiânia, v. 42, n. 2, p. 241-245, 2012. DOI: http://dx.doi.org/10.1590/S1983-40632012000200016

TAVARES-JÚNIOR, J. E.; FAVARIN, J. L.; DOURADONETO, D.; MAIA, A. H. N.; FAZUOLI, L. C.; BERNARDES, M. S. Análise comparativa de métodos de estimativa de área foliar em cafeeiro. Bragantia, Campinas, v. 61, n.2, p. 199-203, 2002.

TOEBE, M.; BRUM, B.; LOPES, S. J.; CARGNELUTTI FILHO, A.; SILVEIRA, T. R. Estimativa da área foliar de Crambe abyssinica por discos foliares e por fotos digitais. Ciência Rural, v. 40, n. 2, p. 475-478. 2010. DOI: http://dx.doi.org/10.1590/S0103-84782010000200036

ZANON, A. J.; STRECK, N. A.; RICHTER, G. L.; BECKER, C. C.; ROCHA, T. S. M.; CERA, J. C.; WINCK, J. E. M.; CARDOSO, A. P., TAGLIAPIETRA; E. L.; WEBER, P. $S$ Contribuição das ramificações e a evolução do índice de área foliar em cultivares modernas de soja. Bragantia, Campinas, v. 74, n. 3, p.279-290. 2015. DOI: http://dx.doi.org/10.1590/1678-4499.0463 